\title{
Are land degradation and desertification worsening in Northern China? Quantitative evidence and enlightenment from satellites
}

\author{
Zecheng Guo ${ }^{1}$, Yaowen Xie ${ }^{2}$, Hui Guo ${ }^{1}$, Xueyuan Zhang ${ }^{1}$, Xihuang Zhang ${ }^{1}$, Guilin Xi ${ }^{1}$, \\ Changhui $\mathrm{Ma}^{1}$, and Hanming Duan ${ }^{1}$ \\ ${ }^{1}$ Lanzhou University \\ ${ }^{2}$ Lanzhou Univ
}

February 3, 2022

\begin{abstract}
Land degradation and desertification (LDD) has become one of the most urgent global environmental issues. The complexity of LDD make it difficult to quantify, how to monitor quickly and accurately has become the key to realize the sustainability for land resources. To achieve this target, firstly, a comprehensive index-land degradation and desertification status index (LDDSI) is built, which integrates the information in fractional vegetation cover (FVC), net primary productivity (NPP), albedo and modified temperature vegetation drought index (MTVDI) based on the spatial principal component analysis (SPCA). Then, identifies LDD from dynamics of land degradation and desertification status (LDDS) in 2001-2018. Based on this, we analyze the spatio-temporal process and driving mechanism of LDD in Northern China. The result indicates that: (a) LDDSI has a better monitoring performance, (b) LDD has been effectively alleviated, but the spatial distribution of LDDS maintains a high clustering pattern, which is difficult to be broken, (c) LDD in local regions is further expanded (1.75\%) affected by many factors, which deserves our attention, and (d) the differences in climate, environmental backgrounds and human activities play a key role in LDDS and LDD. In addition, we assess the effectiveness of ecological projects implemented by the Chinese government. The current understanding in the change pattern and influencing mechanism for LDDS and LDD can provide a scientific basis for formulating ecological policies based on local conditions.
\end{abstract}

\section{Hosted file}

Manuscript.docx available at https://authorea.com/users/458800/articles/555264-are-landdegradation-and-desertification-worsening-in-northern-china-quantitative-evidence-andenlightenment-from-satellites

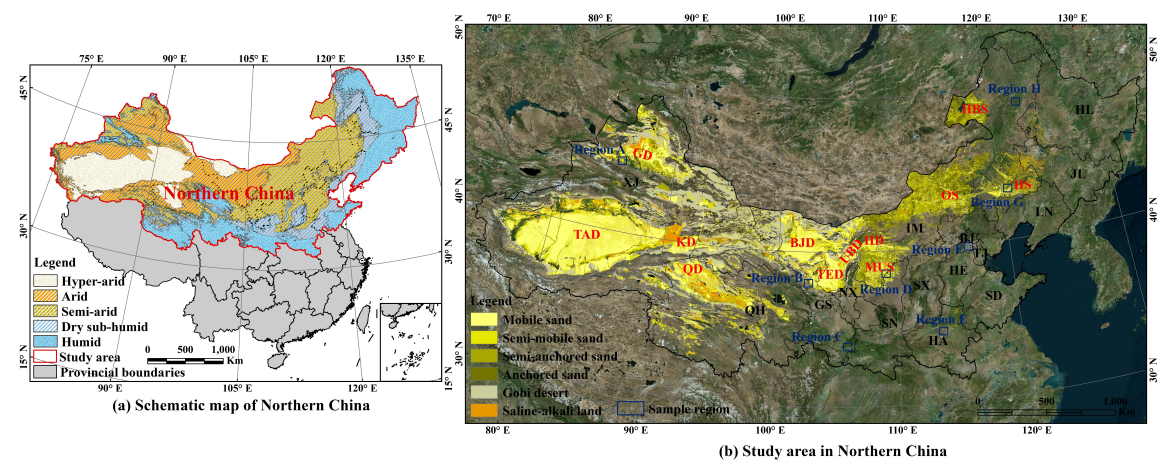



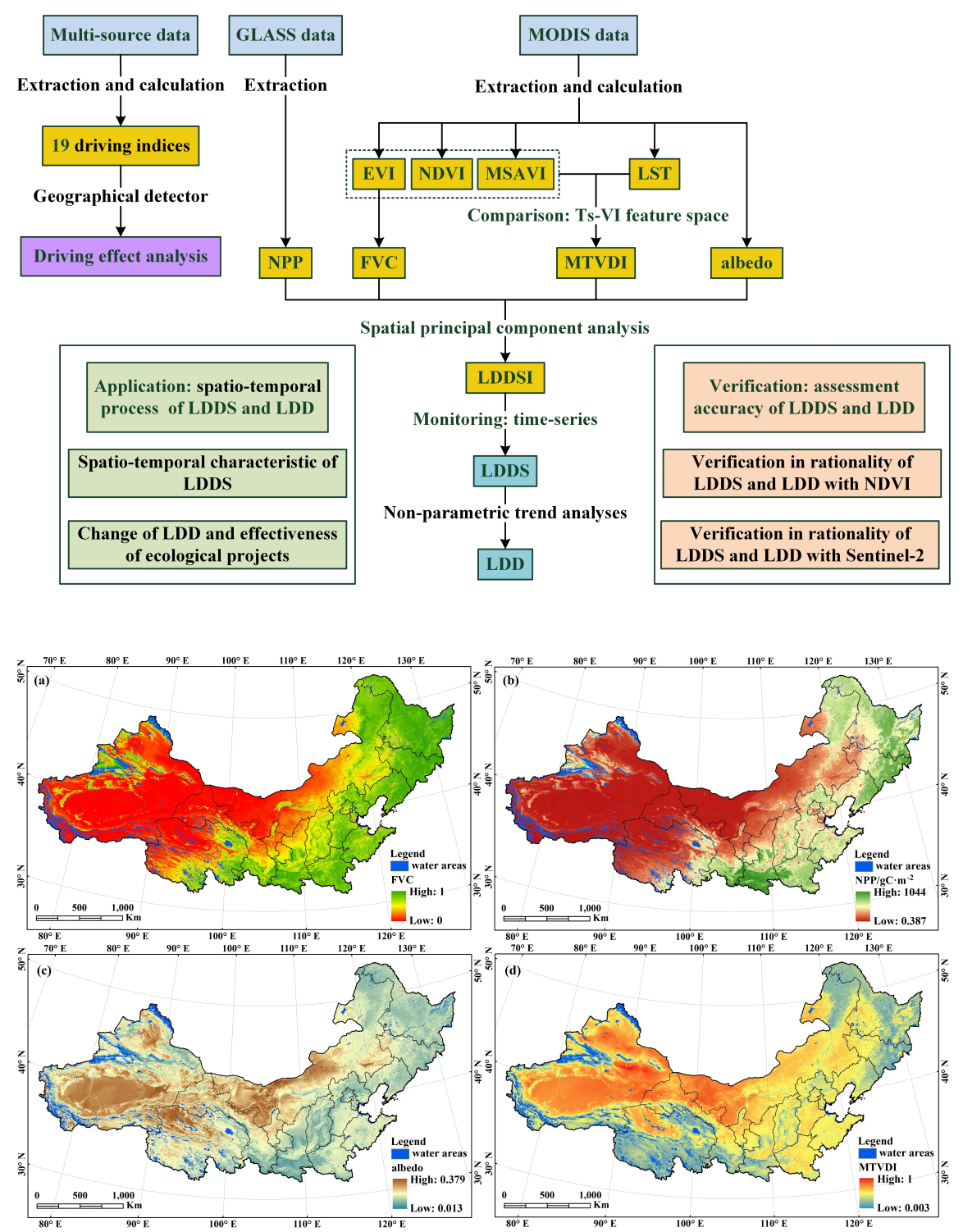

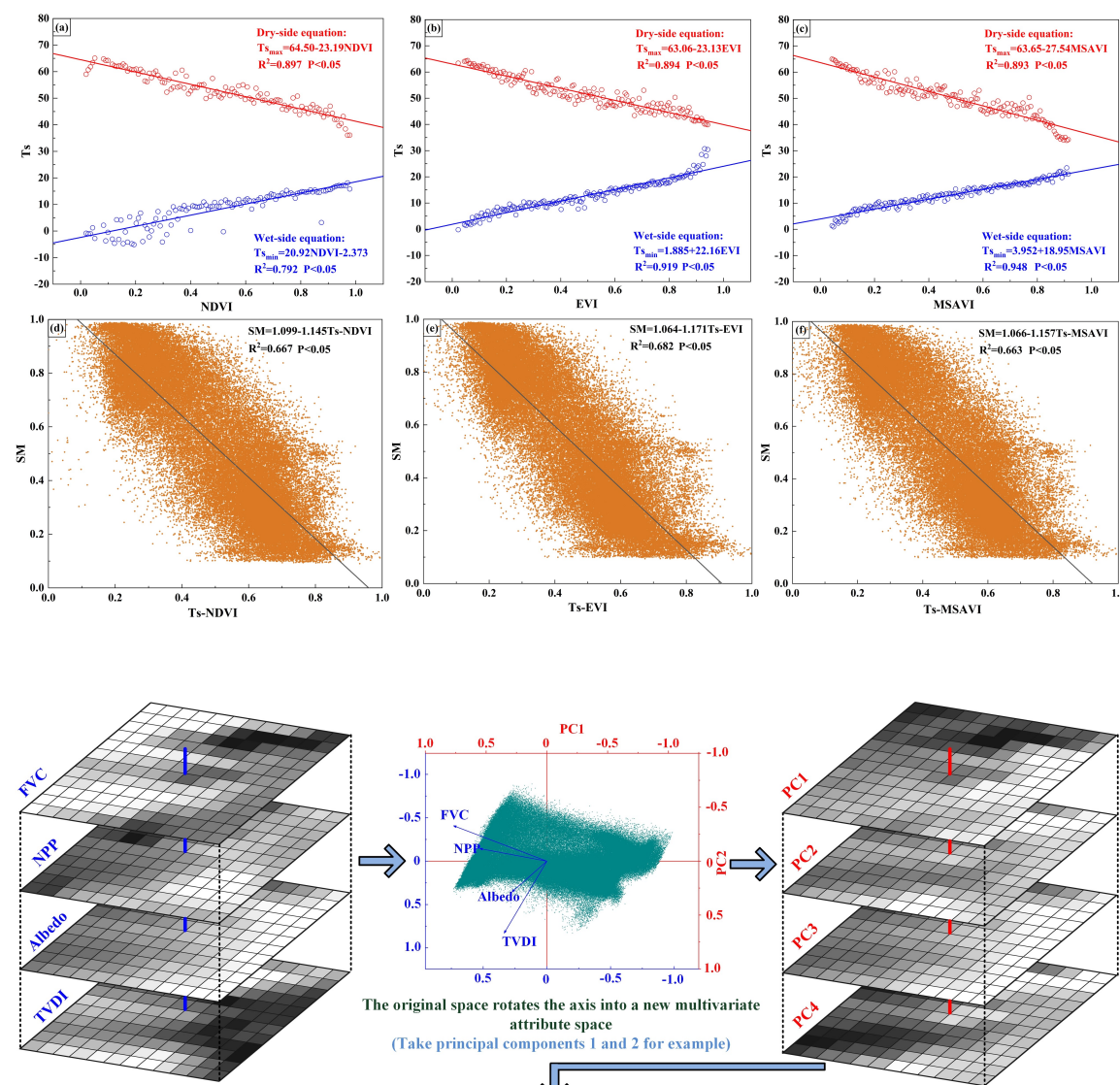

The original space rotates the axis into a new multivariate attribute space
(Take principal components 1 and 2 for example)

Standardized dataset of assessment indices
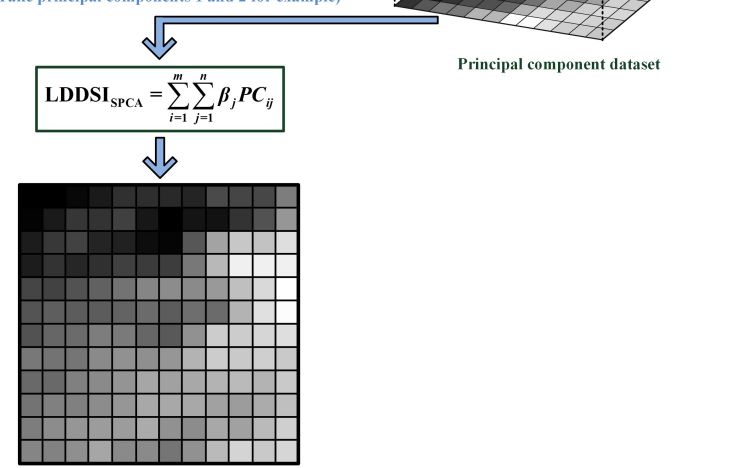

Land degradation and desertification status index (LDDSI) 

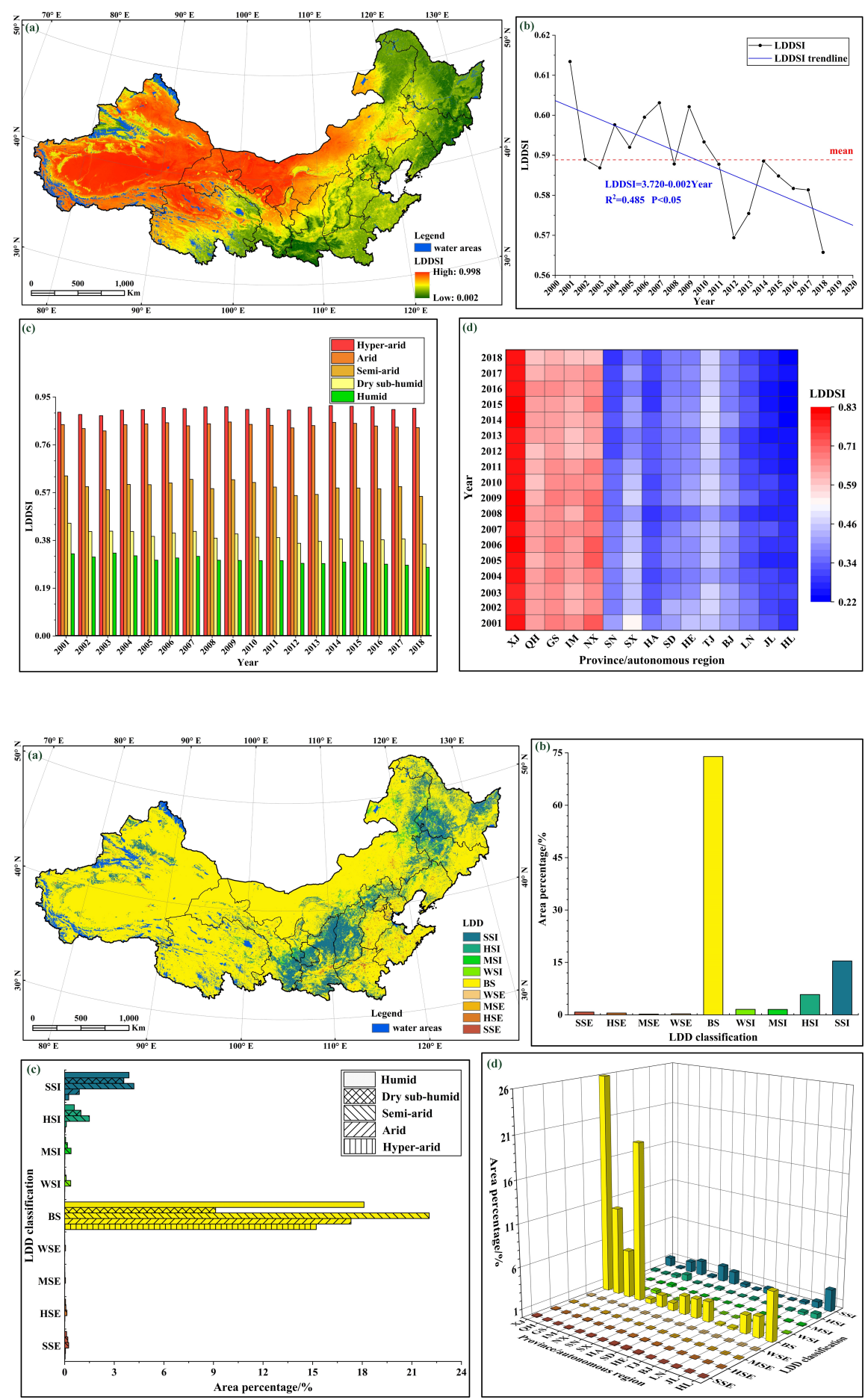

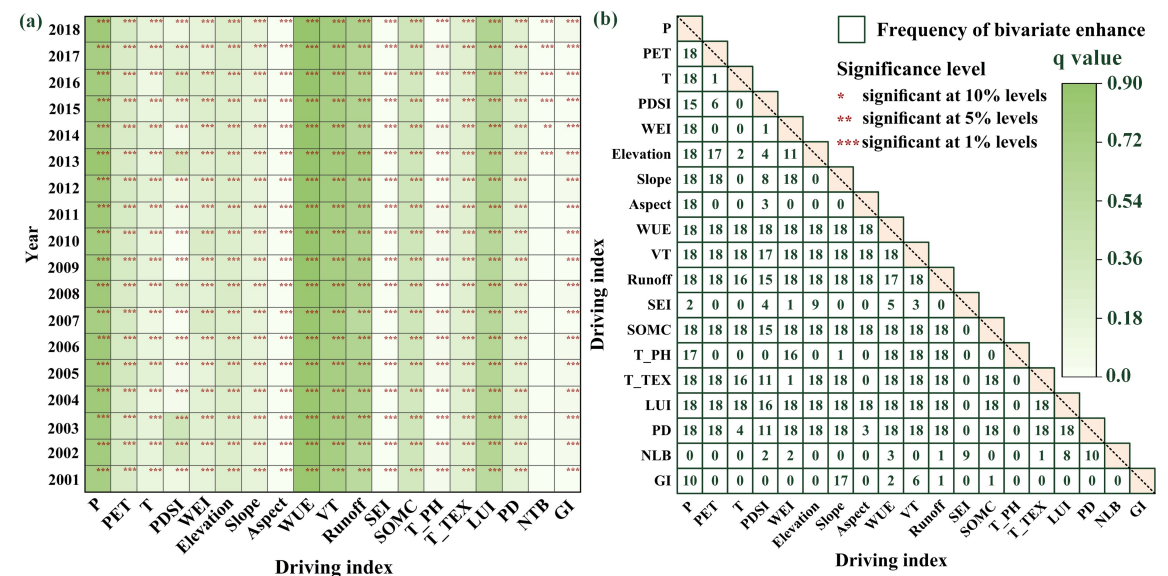

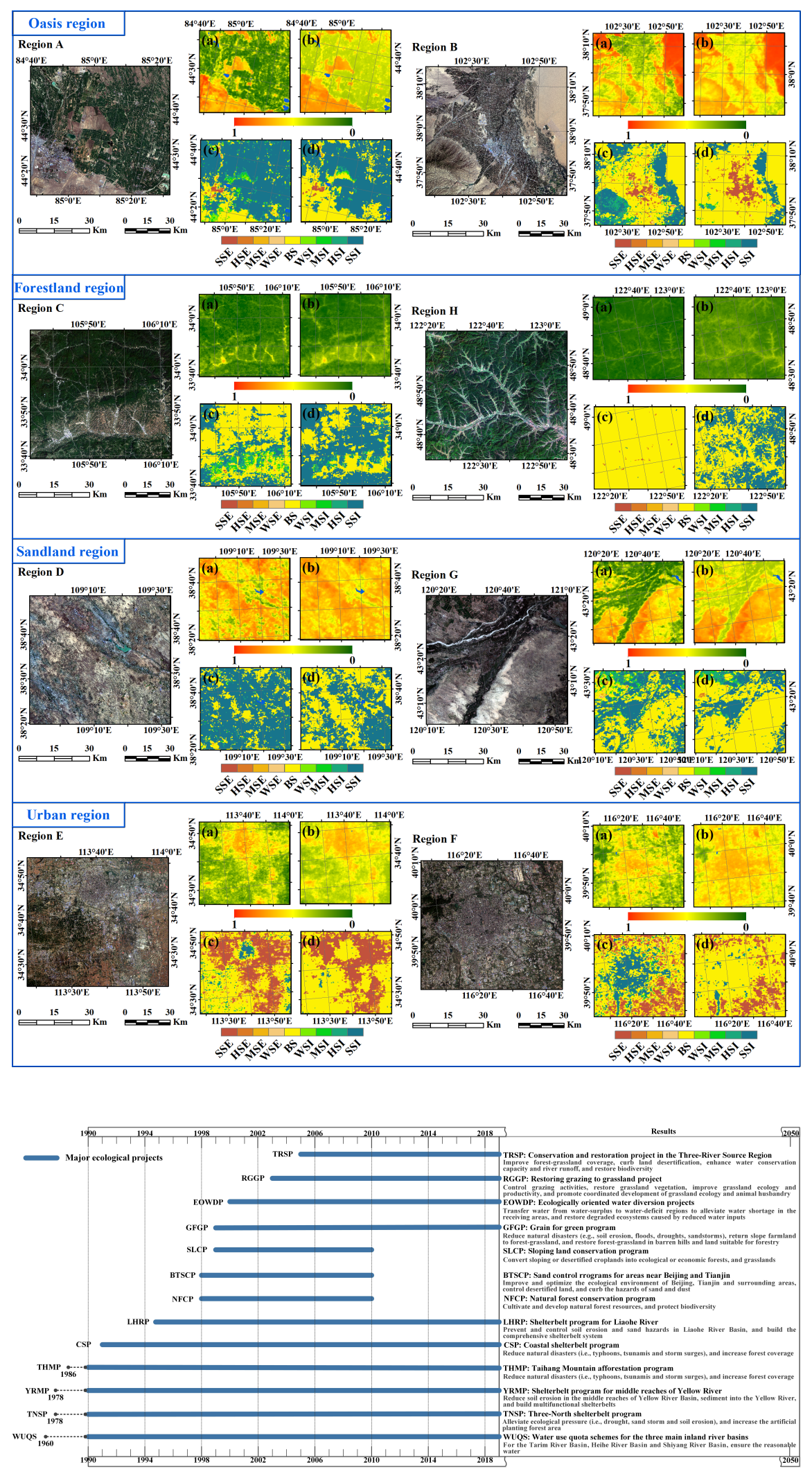